
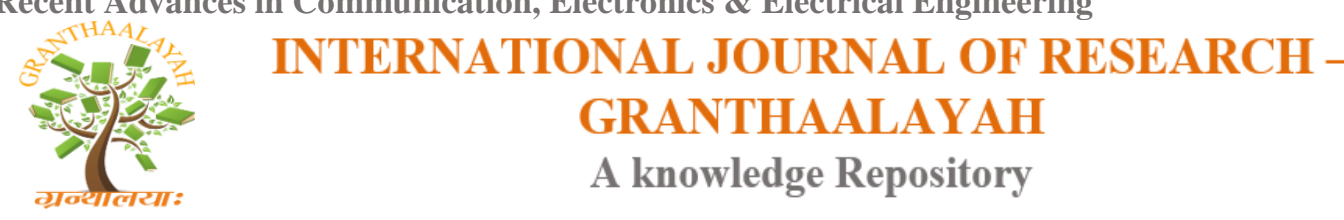

RACEEE - 17

\title{
DESIGN AND PERFORMANCE EVALUATION OF FREE SPACE ENERGY HARVERSTING AND WIRELESS CHARGING
}

\author{
Abhinandan Shetty ${ }^{1}$, Nandish $\mathbf{R}^{2}$, Ruthwik R Soudry ${ }^{3}$, K Sriram ${ }^{4}$, Manjunath K $\mathbf{N}^{5}$ \\ 1,2,3,4 Department of Computer Science and Engineering, School of Engineering and Technology, \\ Jain University, India \\ ${ }^{5}$ Department of Electronics and Communication Engineering, School of Engineering and \\ Technology, Jain University, India
}

DOI: https://doi.org/10.29121/granthaalayah.v5.i4RACEEE.2017.3315

\begin{abstract}
This paper investigates on the wireless charging of a device using the radio frequencies available within the range of $300 \mathrm{MHz}$ to $2.5 \mathrm{GHz}$ in our surroundings. The design is made to store the converted energy in a battery which in turn charges a mobile using inductive charging. This work emphasizes on strengthening the harvested signals from the free space through the design of cascaded voltage multiplier circuit. The main goal of the work is to use renewable energy which is environment friendly and to eliminate the usage of power adapter so that it is easy for charging multiple devices at any time and any place. This paper focuses on improving the previously existing technologies and cutting down the limitations and the idea proposed would be a boon to encourage the use of renewable energy that is environment friendly.
\end{abstract}

Keywords: Rectifier; RF Sources; NI Multisim; Charge Controller; Electro Magnetic Radiation (EMR).

Cite This Article: Abhinandan Shetty, Nandish R, Ruthwik R Soudry, K Sriram, and Manjunath K N. (2017). "DESIGN AND PERFORMANCE EVALUATION OF FREE SPACE ENERGY HARVERSTING AND WIRELESS CHARGING." International Journal of Research Granthaalayah, 5(4) RACEEE, 1-7. 10.29121/granthaalayah.v5.14RACEEE.2017.3315.

\section{Introduction}

Wireless charging has been existing since early 1990's. Wireless charging can be useful for devices like laptops, mobile phones and to the new technology watches. Various wireless devices have taken an edge over the consumer's daily needs. Unfortunately, the present era of wireless technology is constrained by not being able to function as an independent module of power source for a longer duration. There by, the quality of wireless devices being useful, as well as its applications, is severely limited by sluggish improvements in wireless technology. As a result, a 
Recent Advances in Communication, Electronics \& Electrical Engineering prominent technology that allows wireless devices to function for a longer duration and can store electrical energy has to be proposed. There have been researches done in the area of wireless charging to charge electronic devices but none of the design prevails the market that uses renewable energy.

The new technologies focus on decreasing the power consumption and also the size. There by resulting in decrease of power as well as size. Presently, these devices use batteries as their power source. In spite of batteries being considered as means of storage which are not pertaining to the environment, it leads to disadvantages such as short life span of a battery and it needs to be replaced or recharged periodically.

This report embraces the idea of charging mobile devices wirelessly using Ambient RF and inductive charging. The reason for using inductive charging is because direct harvesting of ambient RF to mobile devices takes longer duration. Hence, we store the electrical energy in a rechargeable battery device and transfer the stored electrical energy from the battery device to multiple devices through inductive charging at same time. Ambient backscatter is a technique which uses radio frequency signals, such as radio, television and other RF devices, transmitting data. A device that is used to capture the electromagnetic signals in our surroundings is embedded into a circuit and the power of these signals is used as a power source for some devices or to charge a battery. Device can be named as a charging station, where the device consists of magnetic coil. Power captured from the atmosphere will be stored in the device and it will be in form of $\mathrm{AC}$ but since the cell phone needs the energy in the form of $\mathrm{DC}$ we use rectifier to convert AC to DC. This DC is used in turn for wireless Inductive charging to transfer energy from charging station to Cell phones using electromagnetic field to transfer energy through electromagnetic induction.

\section{Existing Technologies}

The output power obtained relies on the source power as well as distance between the circuit and the RF source (i.e. as distance increases the power generated reduces).In order to implement the proposal, the proposed circuit has to be embedded into a mobile device or a portable device which is not feasible to all the devices. Higher ranges of electromagnetic radiation have been proved unsafe for humans. The risks include tissue deterioration, brain neoplasm and other mental diseases.

Table 1: Energy harvesting sources and their characteristics

\begin{tabular}{|l|l|l|l|}
\hline Energy harvesting sources today & & Harvested power \\
\hline Light & Characteristics & Efficiency & $100 \mathrm{~mW} / \mathrm{cm}^{2}$ \\
\hline Thermal & $\begin{array}{l}\text { Outdoor } \\
\text { Indoor }\end{array}$ & $10 \sim 24 \%$ & $100 \mu \mathrm{W} / \mathrm{cm}^{2}$ \\
\hline Vibration & Human Industrial & $\sim 0.1 \%$ & $60 \mu \mathrm{W} / \mathrm{cm}^{2}$ \\
& $\sim 3 \%$ & $\sim 1-10 \mathrm{~mW} / \mathrm{cm}^{2}$ \\
\hline RF & $\begin{array}{l}\sim \mathrm{Hz}-H u m a n s \\
\mathrm{kHz}-\text { machines }\end{array}$ & $25 \sim 50 \%$ & $\sim 4 \mu \mathrm{W} / \mathrm{cm}^{2}$ \\
\hline
\end{tabular}




\section{Methodology}

Electromagnetic radiation is a self-disseminating wave carrying electromagnetic radiant energy. Radio Waves is one of the important type of electromagnetic radiation with a frequency ranging from $30 \mathrm{~Hz}$ to $300 \mathrm{GHz}$. A device is used along with an antenna to capture the electromagnetic signals in the surroundings and the obtained power is used to recharge the battery. RFID devices are used to capture the RF signals present in the surroundings (that is renewable energy source) and convert this in to the electric power and this electric power is used to charge mobile phones using inductive coupling.

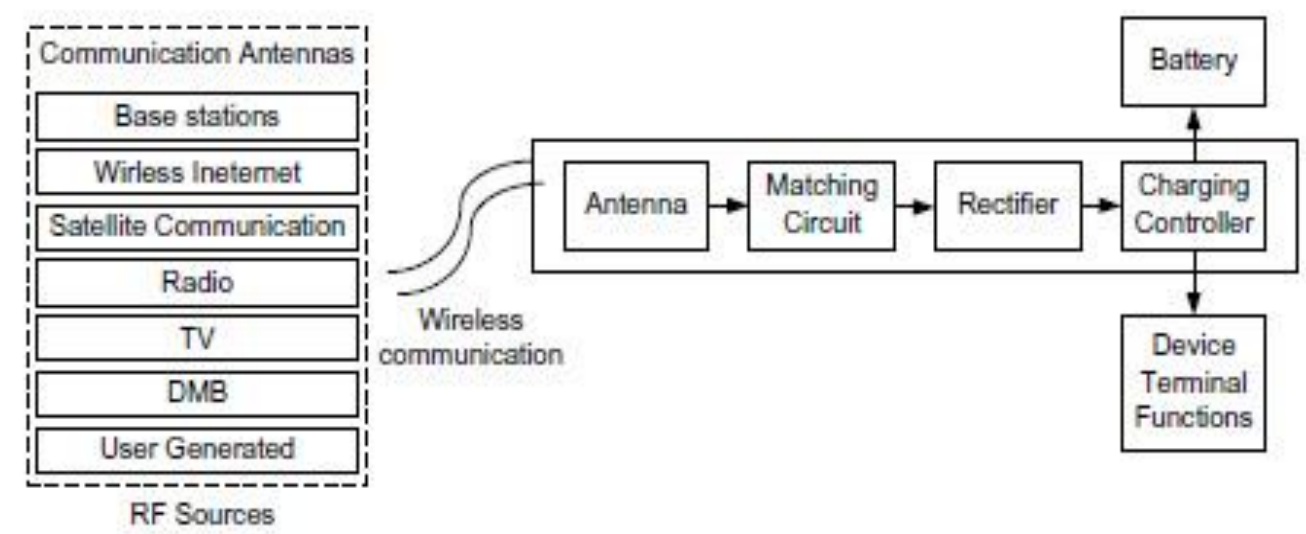

Figure 1: Block diagram for RF energy harvesting

The above diagram shows how the antenna captures the RF signals generated by sources (surroundings). A matching circuit is used along with a rectifier to store DC power in the device. Energy harvesting is performed by using the electromagnetic energy from the surroundings to produce useful electrical energy.

\section{Overview}

The RF in our surroundings generates RF energy. The day to day resources and gadgets such as television, Mobile device, base station, WiFi connections etc. emit electromagnetic energy, Thereby eradicating the usage of RF transmitter as result power, space and energy can be conserved. The RF of interest ranges from $300 \mathrm{MHz}-3 \mathrm{GHz}$ depending on the environment. With the increasing the number of wireless devices, the urban commercial areas encompass these UHF range. In general, in an urban environment the power density ranges from $0.1-0.6 \mathrm{~mW} / \mathrm{m}^{2}$.

\subsection{Development Procedure of Wireless Charger}

The proposed circuit will charge a battery by using the RF signals available in the surroundings (ambient RF). Here the RF signal to DC signal conversion takes place, DC signal is used to charge the battery. 
Recent Advances in Communication, Electronics \& Electrical Engineering

Matching circuit and Antenna: The RF signals are captured using an antenna which is multiband. RF Energy sources are more efficient compared to any other energy sources. The Antenna such as GSM $900 \mathrm{MHz}$ shows the $\sim 50 \%$ of energy efficiency. The harvested power is $0.1 \mu \mathrm{W} / \mathrm{cm}^{2}-$ $0.001 \mu \mathrm{W} / \mathrm{cm}^{2}$. These are usually small in size. At a frequency range of $900 \mathrm{MHz}$ a GSM $50 \Omega$ antenna is used to capture the max power. RF antenna is used to capture the RF signals in our surroundings and it used.

Voltage Rectifier: A rectifier is used to get a DC signal out of the RF which is usually an AC signal. Initially the collected signal is in the form of alternating current so in order to convert it into direct current we use Rectifier.

Voltage Multiplier circuit: Voltage multiplier a common charge pump circuit which is used to amplify the input voltage, by using multiple stages, so that the output voltage obtained is desirable. The output voltage is approximately double the peak input voltage minus two times the threshold voltage of diode used. Due to the charge drain of capacitor the output voltage drops. In order to obtain an output voltage equal to $2 n$ Vin the circuit should be cascaded up to $n$ stages. Diodes are used in order to achieve a low turn on voltage and frequency of operation must be high.

Charge controller circuit: Its primary job is to limit the rate of current being added or drawn from electric batteries. The risk of overcharging can be avoided as overcharging leads to reduction in performance and life span of a battery. It may also help in avoiding deep discharging of a battery,

\subsection{Inductive Charging Using Portable Pad/Case}

The three main components used for wireless power transfer are as follows:

The transceiver transmits the power electromagnetically with the help of inductive coils to the receiver.

Coupling using Induction: With the help of an antenna AC voltage is forwarded to a rectifier where the conversion of $\mathrm{AC}$ voltage to $\mathrm{DC}$ voltage takes place.

Rectification: It is the responsibility of the rectifier to supply the DC voltage to load (mobile device).

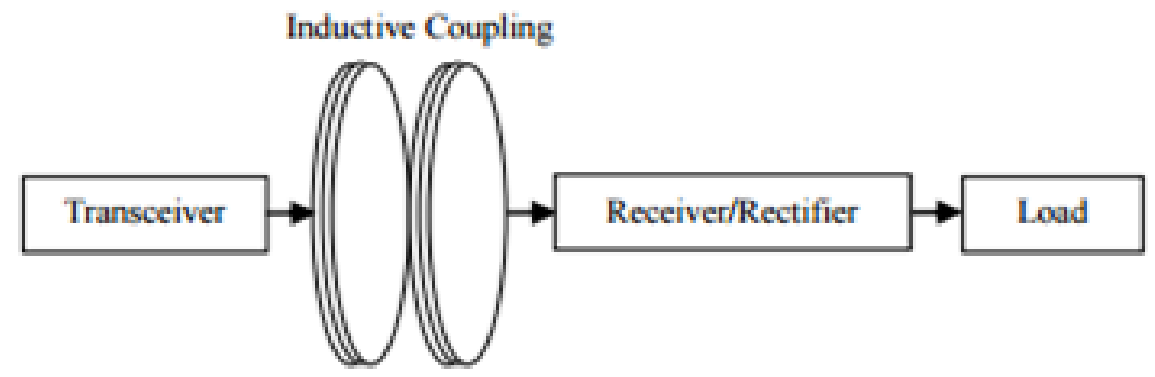

Figure 2: Block diagram for wireless power transfer. 


\subsubsection{Wireless Power Transfer Using Inductive Coupling}

A mobile device is used as a load. The transceiver unit has to be conducted. Energy is transferred to the receiver through electromagnetic induction using inductive coupling. The power source (voltage) for the transceiver is given by the function generator or a voltage source with a frequency of $1.2 \mathrm{MHz}$ and a peak to peak $\mathrm{AC}$ voltage of 0.01 . The inductive coupling is responsible for the wireless power transfer from the transceiver to the receiver. To obtain DC voltage a rectifier is used which converts $\mathrm{AC}$ voltage to $\mathrm{DC}$ voltage. To reduce ripple voltage capacitor is included in the circuit.

\section{Results}

The proposed circuit for two stage and four stage voltage multiplier with matching circuit is simulated on NI Multisim platform. The circuits are as shown in the fig 3 and fig 4 . The current and the voltage values are compared between two stage and four stage voltage multiplier designs. The results show that there is an increase in voltage values as the number of stages increases.

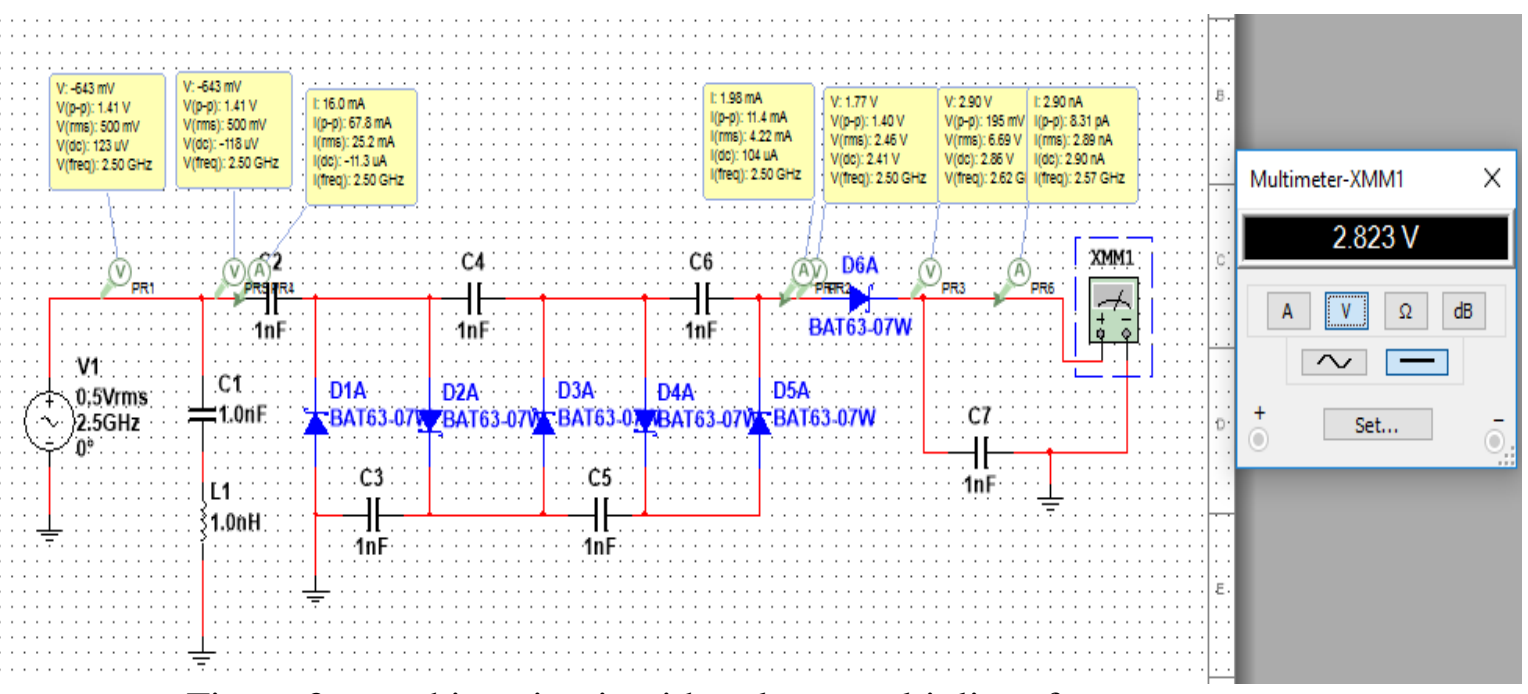

Figure 3: matching circuit with voltage multiplier of two stages.

Table 2: Values obtained for the two stage voltage multiplier.

\begin{tabular}{lll}
\hline $\begin{array}{l}\text { Frequency } \\
\text { (MHz) }\end{array}$ & Voltage(V) & Current (A) \\
\hline $300 \mathrm{MHz}$ & 1.6 & 0.026 \\
$600 \mathrm{MHz}$ & 2.45 & 0.031 \\
$1.2 \mathrm{GHz}$ & 2.823 & 0.033 \\
$2.5 \mathrm{GHz}$ & 2.82 & 0.033
\end{tabular}


Recent Advances in Communication, Electronics \& Electrical Engineering

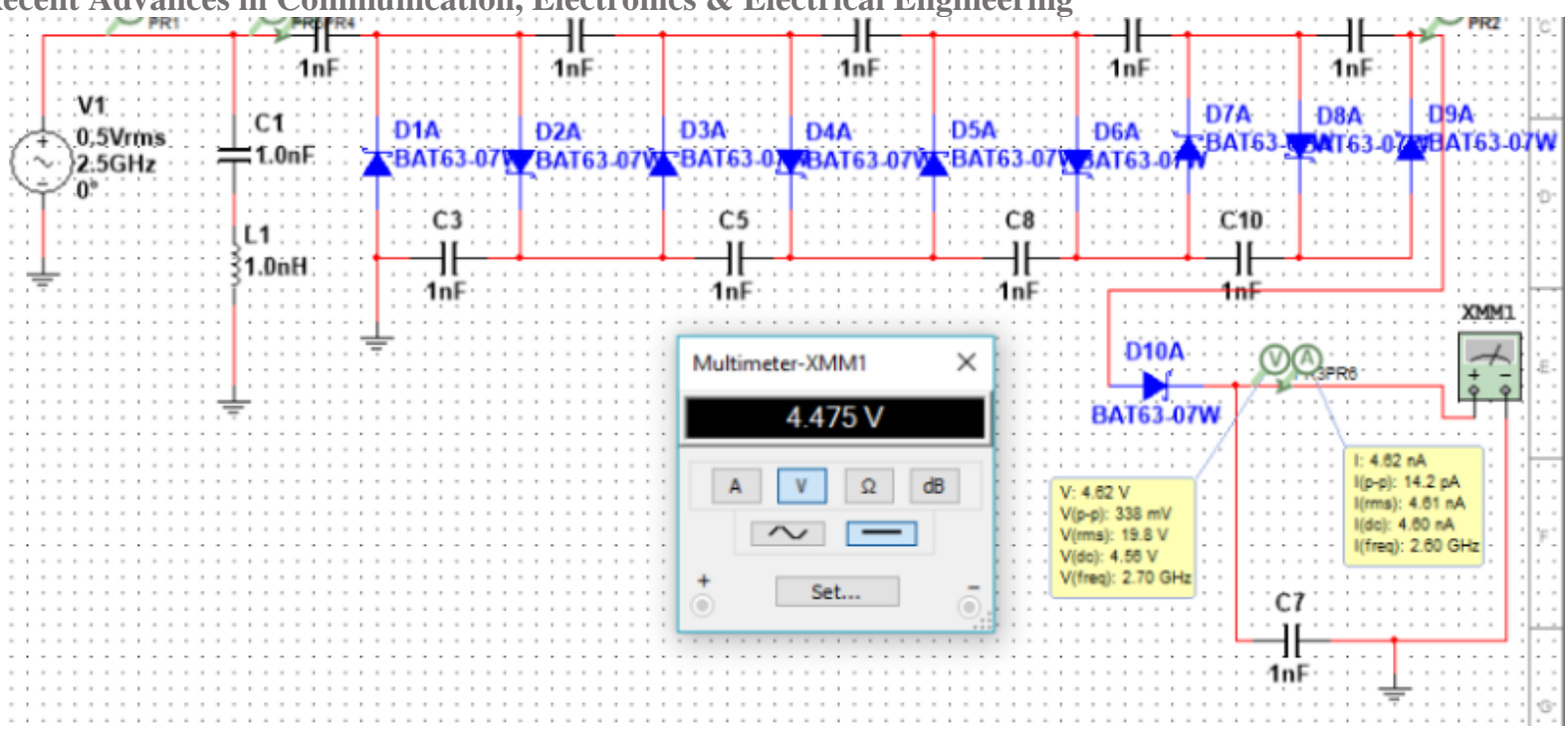

Figure 4: Matching circuit with voltage multiplier of four stages.

Table 3: Values obtained for the two stage voltage multiplier.

\begin{tabular}{lll}
\hline $\begin{array}{l}\text { Frequency } \\
\text { (MHz) }\end{array}$ & Voltage(V) & Current (A) \\
\hline $300 \mathrm{MHz}$ & 3.747 & 0.014 \\
$600 \mathrm{MHz}$ & 4.26 & 0.022 \\
$1.2 \mathrm{GHz}$ & 4.4 & 0.028 \\
$2.5 \mathrm{GHz}$ & 4.475 & 0.031 \\
\hline
\end{tabular}
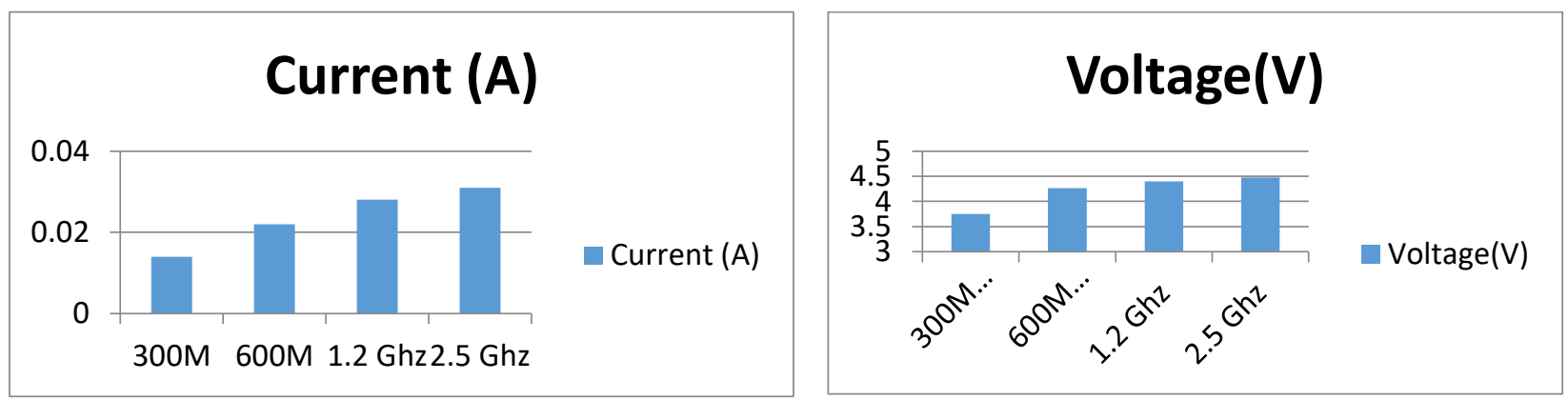

Figure 5: Current and voltage plots of four stage voltage multiplier.

\section{Conclusion}

This work presents the mode of wireless charging by considering RF signals as the naturally available energy source. Here the proposed design is designed and simulated on NI Multisim platform individually by considering the matching circuit and voltage multiplier. The design is simulated for various values of frequencies ranging from $300 \mathrm{MHz}$ to $2.5 \mathrm{GHz}$ and respective current and voltage values are plotted and compared. This work drawn some observation on the change (doubled) of voltage values and remains the current rate. To charge the ideal portable 
Recent Advances in Communication, Electronics \& Electrical Engineering device, the obtained current is not enough and needs to amplify the current with a suitable current amplifier.

\section{Acknowledgements}

The Authors would like thank the management of Jain Group of institutions. The work has been carried out at project laboratory Department of Computer Science and Engineering, School of Engineering and Technology, Jain University.

\section{References}

[1] Hamid Jabbar, Youngseok Song and Taikyeong Ted. Jeong, "Wirelessly Charging Mobile Devices from Ambient RF Sources", Manuscript received January 16, 2010.

[2] Hossam Mahmoud Gamal, ElDin Mohammed ElAnzeery, Mohamed Abd ElAziz Saad ElBagouri and RafikGuindi, "Novel Radio Frequency Energy Harvesting Model" 2012 IEEE International Power Engineering and Optimization Conference (PEOCO2012), Melaka, Malaysia: 6-7 June 2012.

[3] Chen Chen, Xin Wang and Mingyu Lu, "Wireless Charging to Multiple Electronic Devices Simultaneously in Enclosed Box", APEMC 2016.

[4] Anuj kumar, Puneet Kumar Vats, Shoaib Mohammed Ali and B. Kaleeswari, "Energy harvesting From Ambient RF Sources for Mobile Charging", Proceedings of IRF International Conference, 13th April-2014, Chennai, India, ISBN: 978-93-84209-05-6.

*Corresponding author.

E-mail address: abhishetty76@gmail.com 